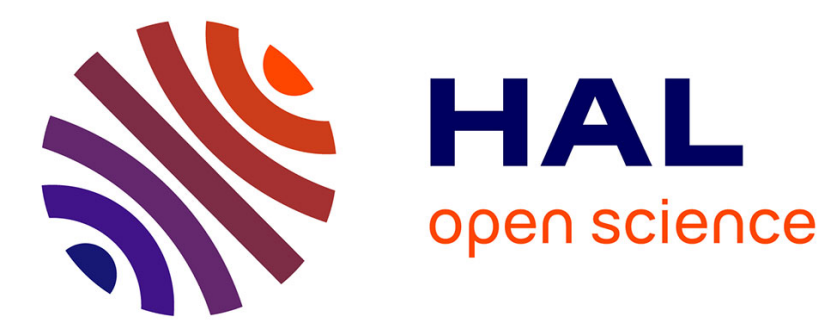

\title{
A CLT for renewal processes with a finite set of interarrival distributions
}

Aurel Spătaru

\section{To cite this version:}

Aurel Spătaru. A CLT for renewal processes with a finite set of interarrival distributions. Statistics and Probability Letters, 2010, 80 (21-22), pp.1680. 10.1016/j.spl.2010.07.012 . hal-00671843

\section{HAL Id: hal-00671843 \\ https://hal.science/hal-00671843}

Submitted on 19 Feb 2012

HAL is a multi-disciplinary open access archive for the deposit and dissemination of scientific research documents, whether they are published or not. The documents may come from teaching and research institutions in France or abroad, or from public or private research centers.
L'archive ouverte pluridisciplinaire HAL, est destinée au dépôt et à la diffusion de documents scientifiques de niveau recherche, publiés ou non, émanant des établissements d'enseignement et de recherche français ou étrangers, des laboratoires publics ou privés. 


\section{Accepted Manuscript}

A CLT for renewal processes with a finite set of interarrival

distributions

Aurel Spătaru

PII:

S0167-7152(10)00206-3

DOI:

10.1016/j.spl.2010.07.012

Reference:

STAPRO 5750

To appear in: Statistics and Probability Letters

Received date: 9 April 2010

Revised date: 13 July 2010

Accepted date: 14 July 2010

Please cite this article as: Spătaru, A., A CLT for renewal processes with a finite set of interarrival distributions. Statistics and Probability Letters (2010),

doi:10.1016/j.spl.2010.07.012

This is a PDF file of an unedited manuscript that has been accepted for publication. As a service to our customers we are providing this early version of the manuscript. The manuscript will undergo copyediting, typesetting, and review of the resulting proof before it is published in its final form. Please note that during the production process errors may be discovered which could affect the content, and all legal disclaimers that apply to the journal pertain. 


\title{
A CLT for renewal processes with a finite set of interarrival distributions
}

\author{
Aurel Spătaru ${ }^{1}$ \\ Institute of Mathematical Statistics and Applied Mathematics, Romanian Academy, \\ Calea 13 Septembrie, nr 13, 76100 Bucharest 5, Romania
}

\begin{abstract}
We prove a central limit theorem for a renewal process based on a sequence of independent non-negative interarrival times whose distributions are taken from a finite set. The result extends the classical central limit theorem obtained by Takács (1956).

Keywords: Renewal process, finitely inhomogeneous random walk, central limit theorem
\end{abstract}

\section{Introduction}

We consider a renewal process $N(t), t \geq 0$, based on a sequence $X_{n}, n \in \mathbb{N}$, of independent non-negative random variables, where the interarrival times $X_{n}, n \in \mathbb{N}$, have a finite number of possible distributions. More precisely, the set $\mathbb{N}=\{1,2, \ldots\}$ is partitioned into $p$ infinite subsets $\mathbb{N}_{1}, \ldots \mathbb{N}_{p}$, and assume there exists a finite collection of random variables $Y_{1}, \ldots, Y_{p}$ such that the distribution of $X_{n}$ coincides with that of $Y_{i}$ whenever $n \in \mathbb{N}_{i}, 1 \leq i \leq p$. In this case, following Durrett, Kesten and Lawler (1991) and Kesten and Lawler (1992), the sequence $S_{0}=0, S_{n}=X_{1}+\ldots+X_{n}, n \in \mathbb{N}$, is called a finitely inhomogeneous random walk. A summary of results on finitely inhomogeneous random walks can be found in Spătaru (2010). Some classical results, of interest for renewal theory, can be generalized to the case $p \geq 2$. (For these generalizations, the assumption $X_{n} \geq 0$ is dropped.) Actually, if 1 Tel.: + 40217716559;

E-mail address: aspataru@rdslink.ro 
$E\left|X_{n}\right|^{s}<\infty, n \in \mathbb{N}$, then $S_{n} / n^{1 / s} \stackrel{\text { a.s. }}{\rightarrow} 0$ for $0<s<1$, while $\left(S_{n}-E S_{n}\right) / n^{1 / s} \stackrel{\text { a.s. }}{\rightarrow} 0$ for $1 \leq s<2$. Consequently, when $1 \leq s<2$ we can only assert that

$$
\liminf _{n} \frac{S_{n}}{n^{1 / s}}=\liminf _{n} \frac{E S_{n}}{n^{1 / s}} \text { a.s., } \limsup _{n} \frac{S_{n}}{n^{1 / s}}=\limsup _{n} \frac{E S_{n}}{n^{1 / s}} \text { a.s. }
$$

This is a generalization of the Kolmogorov-Marcinkiewicz-Zygmund strong law of large numbers. Next, if $E X_{n}^{2}<\infty, n \in \mathbb{N}$, it is not difficult to check that the Lindeberg-Feller central limit theorem becomes

$$
\frac{S_{n}-E S_{n}}{\sqrt{\alpha_{1}(n) \sigma_{1}^{2}+\ldots+\alpha_{p}(n) \sigma_{p}^{2}}} \stackrel{D}{\rightarrow} \Phi,
$$

where $\Phi$ is the standard normal distribution function, and $\alpha_{i}(n)=\# \mathbb{N}_{i} \cap[1, n]$ and $\sigma_{i}^{2}=$ $\operatorname{Var} Y_{i}$ for $1 \leq i \leq p$. Finally, if $E\left|X_{n}\right|<\infty, n \in \mathbb{N}$, and $\tau$ is a finite $\left\{\sigma\left(X_{1}, \ldots, X_{n}\right)\right\}$-time with $E \tau<\infty$, then $E\left|S_{\tau}\right|<\infty$ and

$$
\left(\min _{1 \leq i \leq p} E Y_{i}\right) E \tau \leq E S_{\tau} \leq\left(\max _{1 \leq i \leq p} E Y_{i}\right) E \tau
$$

This extension of Wald's equation can be obtained by arguing as in the classical i.i.d. case $p=1$.

Resting on these generalizations, we can now point out some basic facts concerning the renewal process $N(t), t \geq 0$, defined as $N(t, \omega)=\max \left\{n: S_{n}(\omega) \leq t\right\}, \omega \in \Omega$. (( $\left.\Omega, \mathcal{K}, P\right)$ is the underlying probability space.) Throughout we assume that $0<E Y_{i}=\mu_{i}$ and $E Y_{i}^{2}<\infty$, $1 \leq i \leq p$, and set $m=\min _{1 \leq i \leq p} \mu_{i}$ and $M=\max _{1 \leq i \leq p} \mu_{i}$. Let $\Omega_{0}=\left\{\omega \in \Omega:\left(S_{n}(\omega)-E S_{n}\right) / n \rightarrow 0\right\}$. Then, on account of (1.1),

$$
\liminf _{n} \frac{S_{n}(\omega)}{n}=\liminf _{n} \frac{E S_{n}}{n}=\liminf _{n} \frac{\alpha_{1}(n) \mu_{1}+\ldots+\alpha_{p}(n) \mu_{p}}{n} \geq m>0, \quad \omega \in \Omega_{0},
$$

and so $S_{n}(\omega) \rightarrow \infty, \omega \in \Omega_{0}$. This entails that $N(t, \omega)<\infty$ for all $t \geq 0$ and $\omega \in \Omega_{0}$. Moreover, since each $X_{n}$ is finite-valued, $\lim _{t \rightarrow \infty} N(t, \omega)=\infty, \omega \in \Omega_{0}$. Further, using again 
(1.1), it follows that

$$
M^{-1} \leq \liminf _{t \rightarrow \infty} \frac{N(t, \omega)}{t} \leq \limsup _{t \rightarrow \infty} \frac{N(t, \omega)}{t} \leq m^{-1}, \quad \omega \in \Omega_{0}
$$

The renewal function $U(t)=E N(t), t \geq 0$, is finite-valued, and so right-continuous, also in this new set-up. To see this, choose $a>0$ such that $b=\max _{1 \leq i \leq p} P\left(Y_{i} \leq a\right)<1$. Next, for $t \geq 0$, choose $l \in \mathbb{N}$ such that $a l>t$. For any $j \geq 0$, we have

$$
\begin{aligned}
& P\left(X_{j+1}+\ldots+X_{j+l}>t\right) \geq P\left(X_{j+1}>a, \ldots, X_{j+l}>a\right) \\
& =\left(1-P\left(X_{j+1} \leq a\right)\right) \ldots\left(1-P\left(X_{j+l} \leq a\right)\right)>(1-b)^{l} .
\end{aligned}
$$

Therefore, if $k l \leq n<(k+1) l$, we get

$$
P\left(S_{n} \leq t\right) \leq P\left(S_{k l} \leq t\right) \leq P\left(X_{j+1}+\ldots+X_{j+l} \leq t, 0 \leq j \leq k-1\right) \leq\left(1-(1-b)^{l}\right)^{k} .
$$

Hence, as $N(t)=\sum_{n \geq 1} I\left\{S_{n} \leq t\right\}$, we obtain

$$
U(t)=\sum_{n \geq 1} P\left(S_{n} \leq t\right)=\sum_{k \geq 1} \sum_{k l \leq n<(k+1) l} \leq l \sum_{k \geq 1}\left(1-(1-b)^{l}\right)^{k}<\infty .
$$

Finally, by applying (1.3) and paralleling the proof of the i.i.d. case, it is not difficult to obtain the version of the elementary renewal theorem in the new setting, namely

$$
M^{-1} \leq \liminf _{t \rightarrow \infty} \frac{U(t)}{t} \leq \limsup _{t \rightarrow \infty} \frac{U(t)}{t} \leq m^{-1}
$$

\section{A central limit theorem}

Takács (1956) established the following central limit theorem for the renewal process $N(t), t \geq 0$, corresponding to the i.i.d. case, i.e. $p=1$. If $\mu=E X_{1}$ and $\sigma^{2}=\operatorname{Var} X_{1}$, then

$$
\lim _{t \rightarrow \infty} P\left(\frac{N(t)-t / \mu}{\sigma \sqrt{t / \mu^{2}}} \leq x\right)=\Phi(x), \quad x \in \mathbb{R} .
$$


Formula (2.1) was generalized in several directions by various authors, in which connection see the excellent summary of results on renewal theory in Gut (2009). The next example shows that, depending on the partition of $\mathbb{N}$ and additional conditions, an analogue of (2.1) holds in our setting.

Example 1. Suppose that $\alpha_{i}(n) / n \rightarrow r_{i}, 1 \leq i \leq p$, and put $\mu=r_{1} \mu_{1}+\ldots+r_{p} \mu_{p}$ and $\sigma^{2}=r_{1} \sigma_{1}^{2}+\ldots+r_{p} \sigma_{p}^{2}$. If $\alpha_{1}(n) \mu_{1}+\ldots+\alpha_{p}(n) \mu_{p}-n \mu=o(\sqrt{n})$ as $n \rightarrow \infty$, then, resting on the proof of the classical i.i.d. case, one can see that (2.1) is verified.

Applying (1.2) and assuming that $\alpha_{1}(n) \sigma_{1}^{2}+\ldots+\alpha_{p}(n) \sigma_{p}^{2} \sim n \sigma^{2}$ as $n \rightarrow \infty$ for some $\sigma \in] 0, \infty[$, we extend the central limit theorem to the new set-up. To do this, we need the following lemma.

Lemma 1. Let $f$ be the function whose graph is the polygonal line joining in order the points $\left(E S_{n}, n\right), n \geq 0$. Then, for any $c \in \mathbb{R}$,

$$
\lim _{t \rightarrow \infty} \frac{f(t)}{f(t+c \sqrt{f(t)})}=1 .
$$

Proof. Notice first that

$$
\frac{t}{M} \leq f(t) \leq \frac{t}{m}, \quad t \geq 0
$$

and so

$$
\lim _{t \rightarrow \infty} \frac{\sqrt{f(t)}}{t}=0
$$

(2.4) implies that $\lim _{t \rightarrow \infty}(t+c \sqrt{f(t)})=\infty, c \in \mathbb{R}$. Next, a small modification of the graph of $f$, making $f$ everywhere differentiable, shows that

$$
\frac{f(t)}{f(t+c \sqrt{f(t)})}-1=\frac{-c \sqrt{f(t)}}{f(t+c \sqrt{f(t)})} \cdot a(t)
$$


with $a(t) \leq 1 / m$, whenever $t+c \sqrt{f(t)}>0$. By (2.3) and (2.4), we have

$$
\frac{\sqrt{f(t)}}{f(t+c \sqrt{f(t)})} \leq \frac{\sqrt{\frac{t}{m}}}{\frac{t+c \sqrt{f(t)}}{M}}=\frac{M}{\sqrt{m}} \cdot \frac{1}{\sqrt{t}\left(1+c \frac{\sqrt{f(t)}}{t}\right)} \rightarrow 0 \text { as } t \rightarrow \infty .
$$

Now (2.2) follows from (2.5) and (2.6).

We can now state and prove our main result.

Theorem 1. Let $f$ be as in Lemma 1, and assume that $\alpha_{1}(n) \sigma_{1}^{2}+\ldots+\alpha_{p}(n) \sigma_{p}^{2} \sim n \sigma^{2}$ as $n \rightarrow \infty$ for some $\sigma \in] 0, \infty[$. Then

$$
\lim _{t \rightarrow \infty} P(N(t)>f(t-x \sigma \sqrt{f(t)})=\Phi(x), \quad x \in \mathbb{R}
$$

Proof. Write $P(t, x)=P(N(t)>f(t-x \sigma \sqrt{f(t)}), t \geq 0, x \in \mathbb{R}$. For $n \geq 1$, we have

$$
E S_{n-1} \leq t<E S_{n} \Longleftrightarrow n-1 \leq f(t)<n
$$

For fixed $x \in \mathbb{R}$, we have noticed in the proof of Lemma 1 that $\lim _{t \rightarrow \infty}(t-x \sigma \sqrt{f(t)})=\infty$. Suppose now that $t-x \sigma \sqrt{f(t)}>0$, and let $n=n(t)$ be defined by $E S_{n-1} \leq t-x \sigma \sqrt{f(t)}<$ $E S_{n}$. Observe that $n \rightarrow \infty$ as $t \rightarrow \infty$. By (2.8),

$$
n-1 \leq f(t-x \sigma \sqrt{f(t)})<n
$$

We have $x \sigma \sqrt{f(t)}-E X_{n} \leq t-E S_{n}<x \sigma \sqrt{f(t)}$, and so

$$
x \sqrt{\frac{f(t)}{n}}-\frac{E X_{n}}{\sigma \sqrt{n}} \leq \frac{t-E S_{n}}{\sigma \sqrt{n}}<x \sqrt{\frac{f(t)}{n}} .
$$

Further, we have

$$
P(t, x)=P(N(t) \geq n)=P\left(S_{n} \leq t\right)=P\left(\frac{S_{n}-E S_{n}}{\sigma \sqrt{n}} \leq \frac{t-E S_{n}}{\sigma \sqrt{n}}\right)
$$


In view of (2.10), this implies

$$
P\left(\frac{S_{n}-E S_{n}}{\sigma \sqrt{n}} \leq x \sqrt{\frac{f(t)}{n}}-\frac{E X_{n}}{\sigma \sqrt{n}}\right) \leq P(t, x) \leq P\left(\frac{S_{n}-E S_{n}}{\sigma \sqrt{n}} \leq x \sqrt{\frac{f(t)}{n}}\right) .
$$

On account of $(2.9)$, we see that

$$
\frac{n-1}{n} \cdot \frac{f(t)}{f(t-x \sigma \sqrt{f(t)})} \leq \frac{f(t)}{n}<\frac{f(t)}{f(t-x \sigma \sqrt{f(t)})}
$$

According to Lemma 1, this entails that

$$
\lim _{t \rightarrow \infty} \frac{f(t)}{n}=1
$$

Finally, since $E X_{n} \leq M, n \in \mathbb{N},(2.7)$ follows from (1.2), (2.11) and (2.12).

Remark 1. If $p=1, \mu=E X_{1}$ and $\sigma^{2}=\operatorname{Var} X_{1}$, then $f(t)=t / \mu, t \geq 0$, and (2.7) becomes

$$
\lim _{t \rightarrow \infty} P\left(N(t)>\frac{t}{\mu}-x \sigma \sqrt{\frac{t}{\mu^{3}}}\right)=\Phi(x), \quad x \in \mathbb{R}
$$

Thus (2.1) is a special case of (2.7).

Remark 2. Theorem 1 applies with $\sigma^{2}=r_{1} \sigma_{1}^{2}+\ldots+r_{p} \sigma_{p}^{2}$ whenever $\alpha_{i}(n) / n \rightarrow r_{i}, 1 \leq i \leq p$.

Remark 3. An inspection of the proof of Theorem 1 shows that (2.7) holds true whenever $X_{n}, n \in \mathbb{N}$, is an arbitrary sequence of random variables with $0<\inf _{n \in \mathbb{N}} E X_{n} \leq \sup _{n \in \mathbb{N}} E X_{n}<\infty$ such that $\left(S_{n}-E S_{n}\right) / \sigma \sqrt{n} \stackrel{D}{\rightarrow} \Phi$ for some $\left.\sigma \in\right] 0, \infty[$.

\section{Acknowledgment}

The author is grateful to the referee for suggestions that improved the presentation.

\section{References}

Durrett, R., Kesten, H., Lawler, G., 1991. Making money from fair games, in: R. Durrett, H. Kesten, eds. Random Walks, Brownian Motion and Interacting Particle Systems. Birkhäuser, Boston, pp. 255-268. 
Gut, A., 2009. Stopped Random Walks, second ed. Springer, New York.

Kesten, H., Lawler, G., 1992. A necessary condition for making money from fair games. Ann. Probab. 20 855-882.

Spătaru, A., 2010. The law of the iterated logarithm for finitely inhomogeneous random walks. J. Theoret. Probab. 23 417-427.

Takács, L. 1956. On a probability problem arising in the theory of counters. Proc. Camb. Phil. Soc. 32 488-498. 